\title{
A Novel Combined Approach for Daily Electric Load Forecasting Based on Artificial Neural Network and Modified Bat Algorithm
}

\author{
Eduardo Capra \\ The University of São Paulo \\ São Carlos, Brazil
}

\author{
Hugo Ribeiro \\ The University of São Paulo \\ São Carlos, Brazil
}

\begin{abstract}
In this paper a novel combined method based on Modified Bat Algorithm (MBA) and Neural Network algorithm has proposed in order to forecast the electric peak load power. In the proposed method, Bat Algorithm is employed as a popular optimization method and Artificial Neural Network is also utilized as a powerful mathematic method in mapping nonlinear relationship among model variables for the purpose of electric daily load prediction. Additionally, in order to improve the performance of the bat algorithm with regards to avoiding tapping into the local optimal and increasing the convergence speed, some modification has performed in bat algorithm which is called as SAMBA. Experimental results indicate that the proposed method has superiority performance in comparison with other traditional machine learning algorithms
\end{abstract}

Keywords: Short-Term Load Forecasting, Bat Algorithm, Artificial Neural Network, Intelligent Systems, Optimization Algorithm

\section{INTRODUCTION}

Electrical energy is considered as one of the mostly used forms of energy due to several benefits that can provide e.g. economical and clean type of energy [1], efficient transmission [2], easy distribution [3], availability [4], etc. Therefore, the demand for electrical energy has become an important issue in such a way that how this type of energy can be distributed and provided to the society efficiently [5-6]. Electrical load forecasting is defined as an intelligent process that predict required electrical power for short-term, mediumterm, and long-term demand [7]. Designing the precise prediction model is essential in order to provide efficient and reliable electrical energy for all consumers. This demand has attracted the attention of scientific researcher to develop an accurate and efficient model for load forecasting [8-11].

Short-term load forecasting is categorized as a prediction of an hour up to a day ahead. Medium-term is considered for a seasonal prediction e.g. summer, winter, etc. additionally, it is used for fuel supplies scheduling for several days up to weeks. And Long-term Forecasting is generally used to make plan for growth of the required generating capacity and transmission that needs a prediction time from few months to few years [12].

In this paper our main focus is for short-term load forecasting by using machine learning algorithms. To this end, we combined both Bat algorithm as an optimization algorithm along with Artificial Neural Network (ANN) to provide an efficient and accurate model for the short-term time domain. In the following section, it will be demonstrated that the level of uncertainty which exists in the load forecasting problem is decreased noticeably. Recently, intelligent systems and machine learning algorithms have been widely used in different range of engineering practical problems. To name a few: using intelligent and expert method as system compensator and optimization method [13-14], modeling adaptive controller for industrial applications [15-16]. In particular, in the area of electric load forecasting, there are many methods available in the literature. In [17] authors proposed a new method that leverage the capability of chaos time series analysis to capture the electric load behavior for the short-term time horizon and then the nonlinear mapping of deterministic chaos is formulated by using perceptron algorithm. There are some other studies that employed time series analysis for load forecasting. [18-19]. In [20] a new method based on fuzzy regression analysis is introduced for short-term load forecasting error for 24 hours during the holidays. Authors believed that the average load forecasting error can be higher compared to the other days during week. In [21] an adaptive autoregressive moving-average model is introduced for electric load prediction and the superiority of their method is compared with the traditional Box-Jenkins transfer function approach. Recently, it is shown that Artificial Neural Network (ANN) has a potential capability of mapping nonlinear variables which are existed in the electric load data. Hence, it has caught the attraction of researcher in this area. In [22] authors present a hybrid model based on Radial Basis Function (RBF) neural network with adaptive neural fuzzy inference system. In this combined approach, the RBF network is firstly used to establish a model for load forecasting without considering the price change and then adaptive neural fuzzy inference system (ANFIS) is used by considering the recent real-time price change to adapt the results of load prediction which has attained by RBF previously. Emerging of evolutionary algorithms have gained great importance over the other existing techniques to adjust the different parameters of intelligent models due to their consistent and robust performance. [23-24]. There are many available evolutionary-based optimization techniques available in the literature for electric load forecasting namely Genetic Algorithm (GA), Particle Swarm Optimization (PSO), Bat Algorithm (BA) [25-26], Crew Search Algorithm (CSA) [27], etc. In [28] the Artificial Neural Network is applied for hourly prediction of load forecasting and particle swarm optimization algorithm is utilized to tune the ANN weights and adjusting factor in the training phase. In [29] Genetic Algorithm is employed to find the most optimal parameters of Support Vector Regression (SVR) to enhance the accuracy and performance of SVR. Although GA is considered as powerful evolutionary-based optimization algorithm, the dependency of this method on its parameter can create a problem of trapping in the local optima. PSO can also face two similar shortcomings: dependency of initial values and trapping in local optima. 
As it was mentioned previously, the proposed method made up of Artificial Neural Network (ANN) and modified Bat algorithm. The method follows these two steps: Firstly, the ANN is trained based on traditional training algorithm e.g. backpropagation and then the most suitable ANN architecture which includes number of input layers, number of hidden layers, number of output layer, number of neurons in each corresponding layer, adjusting and weighting factors. Secondly, a modified Bat optimization algorithm is employed to figure out the most optimized parameters for adjusting and weighting factors of ANN structure. Bat Algorithm can also have the limitations which we discussed earlier for GA and PSO (local optima trapping and premature convergence). In order to prevent algorithm to be trapped in local optima and increase convergence maturity, a new modification is introduced for BA. The main contribution of this paper is given as follows: 1) Finding optimal parameter of ANN based on modified Bat algorithm after the network being trained by classical methods. 2) applying the proposed method for prediction of electric load for the upcoming day.

\section{Multi-Layer Feedforward Neural Network}

Artificial Neural Network is considered as a popular and powerful machine learning method for classification and prediction which is inspired by biological system of human's brain. One of the mostly used application of this method is for forecasting. There are several distinctive features that make ANN as a powerful technique for forecasting (particular for electric load forecasting) e.g. mapping non-linear relations of variables. In general, multi-layer feedforward neural network consists of input layer, one or multi-level of hidden layer, and output layer. There are neurons in each layer and the connection of these layers are done by adjusting and weighting factors. Shown in figure 1 is an example of ANN with two levels hidden layer.

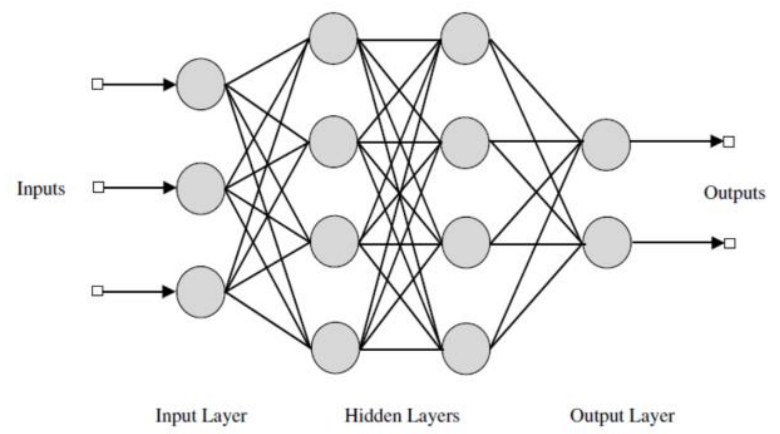

Figure 1. high-level structure of Artificial Neural Network

The determinations of ANN pattern include number of input layers, number of hidden layer, and number of output layer. The number of neurons which are used in input and output layer determine the number of input and output variables, respectively. There is a transfer function in ANN to map the input and output variables. In this paper, the sigmoid transfer function is chosen. Eq. 1 defines the output of each node in ANN.

$$
y_{i}=f_{1}\left(\sum_{j=1}^{N_{h}}\left(w_{i, j}^{2} f_{2}\left(\sum_{k=1}^{N_{i}} w_{j, k}^{1} x_{k}+b_{j}^{1}\right)+b_{i}^{2}\right)\right.
$$

$f_{1:}$ input layer transfer function.

$f_{2}$ : hidden layer transfer function.

$b^{1}$ : hidden layer biasing metric.

$b^{2}$ : output layer biasing metric.

$w^{1}{ }_{i, j}$ weighting parameter to connect input node $(i)$ to the node (j) in hidden layer.

$w^{2} i, j$ weighting parameter to connect hidden layer node $(i)$ to the node $(j)$ in output layer.

\section{Bat Algorithm}

Bat Algorithm is inspired by the echolocation behavior of bats and considered as a new nature-inspired metaheuristic optimization algorithm [30]. This algorithm is inspired by this behavior of bats which they emit sound pulse and listen to the echo that is returning back from obstacles. Bat algorithm is formulated based on below for steps [31]:

1) Bats use echolocation behavior to know the distance of obstacles and figure out the difference between food and prey.

2) Each bat located in the position of $X_{i}$ in the search space and flies with the velocity of $V_{i}$ and emitting a particular pulse with the frequency and loudness of $f_{i}$ and $A_{i}$ respectively.

3) The loudness of $A_{i}$ can be varied in many ways like by decreasing from a large value to a low value

4) The frequency $f_{i}$ and rate $r_{i}$ of each particular pulse is regulated automatically.

Having had a random fly, the location of each bat will be updated as follows:

$$
\begin{aligned}
& \mathbf{V}_{i}^{\text {new }}=\mathbf{V}_{i}^{\text {old }}+f_{i}\left(\text { Gbest }-X_{i}\right) ; i=1, \ldots, N_{B a t} \\
& X_{i}^{\text {new }}=X_{i}^{\text {old }}+\mathbf{V}_{i}^{\text {new }} \quad ; i=1, \ldots, N_{B a t} \\
& f_{i}=f_{i}^{\min }+\varphi_{1}\left(f_{i}^{\max }-f_{i}^{\min }\right) \quad ; i=1, \ldots, N_{B a t}
\end{aligned}
$$

Where:

Gbest: the best bat from the objective perspective.

$N_{\text {Bat }}$ : total number of bat population.

$\varphi_{1}$ : random value in the range of $[0,1]$.

$f_{i}^{\max } / f_{i}^{\min }:$ maximum/minimum frequency values of the $i^{\text {th }}$ bat, respectively.

In order to receive the better random walking, the random number $\beta$ is generated and if the new randomly generated number $\beta$ is greater than $r_{i}$, the new solution is created as follows:

$$
X_{i}^{\text {new }}=X_{i}^{\text {old }}+\varepsilon A_{\text {mean }}^{\text {old }} ; i=1, \ldots, N_{\text {Bat }}
$$

$\varepsilon:$ random value in the interval of $[-1,1]$

$A_{\text {mean }}^{\text {old }}$ : mean value of loudness of all bats.

Where: 
If the randomly generated value $\beta$ is less than $r_{i}$, new position is generated randomly. It should be noted that the following condition should be met in order to accept the new position.

$$
\left[\beta<A_{i}\right] \&\left[f\left(X_{i}\right)<f(\text { Gbest })\right]
$$

Additionally, the loudness and rate of each pulse is updated as follows:

$$
\begin{aligned}
& A_{i}^{\text {new }}=\alpha A_{i}^{\text {old }} \\
& r_{i}^{\text {Iter }+1}=r_{i}^{0}[1-\exp (-\gamma \times \text { Iter })]
\end{aligned}
$$

where $\alpha$ and $\gamma$ are constant values and Iter is the number of the iteration during the optimization process.

\subsection{Modified Bat Algorithm}

As discussed earlier, in order to enhance the overall capability of Bat algorithm to prevent algorithm to be trapped in the local optima and premature convergence. The proposed modification includes two methods:

\section{First modification:}

In Eq. 5 the value of $\alpha$ will be updated during the optimization procedure by following formula:

$\alpha^{\text {new }}=(1 / 2 \text { Iter })^{1 / \text { Iter }} \alpha^{\text {old }}$

\section{Second Modification:}

This modification has introduced in order to increase the diversity of total bat population.

$$
\begin{aligned}
& X_{i}^{\text {new }}= \begin{cases}x_{i, j}^{\text {old }} & ; \varphi_{1}<\varphi_{2} \\
\text { gbest }_{j} & ; \varphi_{1} \geq \varphi_{2}\end{cases} \\
& X_{i}^{\text {new }}=\left[x_{i, 1}^{\text {new }}, x_{i, 2}^{\text {new }}, \ldots, x_{i, n}^{\text {new }}\right]
\end{aligned}
$$

$\varphi_{2}$ : random value between the range of $[0,1]$.

$n$ : length of the control vector.

In the beginning of the procedure, each modification method has an equal probability $\left(\operatorname{Prob}_{\omega}=0.5 \& \omega=1,2\right)$. Each bat selects the $\omega^{\text {th }}$ modification method by using roulette wheel mechanism (RWM). Hence, bats population is sorted out after each loop and the probability of each modification is updated as follows:

$$
\operatorname{Prob}_{\omega}=S R_{\omega} / \sum_{\omega=1}^{2} S R_{\omega} ; \omega=1, \ldots, 2
$$

$S R_{\omega}$ : $\quad$ successful rate of $\omega^{\text {th }}$ modification method for generating optimal bats within the population. The criteria to be more optimal for each bat is defined such that if each new bat's objective function is less than the previous iteration. Having updated the probability of each modification, $\varphi_{3}$ is created as a random number in the range of $[0,1]$. The procedure of selecting the proper modification method $\omega^{\text {th }}$ will be chosen by using roulette RWM to create the next bat population generation.

\section{Proposed Combined Method Based-on ANN and Modified Bat Algorithm:}

In order to leverage the capability of the modified bat algorithm to adjust the ANN parameters, each member of the bat society $\bar{X}_{i}$ includes both weight and biasing factor of ANN which is defined in below equation:

$$
\bar{X}_{i}=\left[w_{i, 1}, \ldots, w_{i, N w}, b_{i, 1}, \ldots, b_{i, N b}\right]_{(1, N w+N b)}
$$

Bat society size is the same as the total number of all weighting and biasing factors of ANN. In order to demonstrate the accuracy and performance of the proposed method, two performance measurement criteria are introduced: relative error and Mean Absolute Percentage Error (MAPE) which are formulated as follows, respectively:

$\sigma_{i} \%=\frac{\left|P_{i}-T_{i}\right|}{T_{i}} \times 100, i=1,2, \ldots, N_{e s}$

$M A P E \%=\frac{1}{N_{e s}} \sum_{i=1}^{N_{e s}} \sigma_{i}$

$P_{i}$ : estimated value of the $i^{\text {th }}$ data.

$T_{i}$ actual value of the $i^{\text {th }}$ data.

$N_{e s}$ : number of data that supposed to be forecasted.

Generally, the less MAPE value, the more accurate forecasting results will be attained. Therefore, the objective function of the proposed method should minimize MAPE value. The procedure of the proposed method is summarized as follows:

Step 1: Generating required parameters: All the input parameters such as number of the input neurons, number of output neurons, training algorithm, bat population size, the initial value, maximum and minimum value for loudness pulse of each bat should be initialized.

Step 2: Data normalization: In order to have a satisfactory results and structure for ANN, data normalization is required.

Step3: Weighting and biasing factors optimization: In this step the proposed method is employed to optimize both weight and biasing factors in ANN. The objective function for this purpose is to keep the MAPE values as minimum.

Step 4: Generating initial population for Bat algorithm: the bat population vector is described as follows:

Bat_population $=\left[\begin{array}{c}X_{1} \\ X_{2} \\ \cdots \\ X_{N_{B a t}}\end{array}\right]_{N_{B a t} \times\left(N_{w}, N_{b}\right)}$

$N_{b a t}$ is defined as the number of whole bats.

Step 5: Objective function measurement: In this step MAPE criterion will be assessed for all bats in population. 
Step 6: Defining the best bat (Gbest): The best $X_{i}$ is chosen as a bat with the least value of MAPE as objective function.

Step 7: Apply the proposed modification bat algorithm to enhance the population diversity.

Step 8: evaluate the termination criteria: if the termination criteria has not been met, go back to step 5. Otherwise, the algorithm will be terminated.

\section{Experimental Results and Evaluations}

In order to evaluate the accuracy and performance of the proposed method and demonstrate the superiority of the proposed method compared to the other evolutionary-based method and traditional methods, a real dataset of electricity consumption of .... in Brazil has been used. This data-set which is used to train the ANN is gathered from March 2008 to March 2016. To this end, the load forecasting method is applied to predict the upcoming day. The input and output features of ANN is summarized in Table 1. In this research, the structure of ANN is defined as ten input features, one output and two hidden layers. It should be noted that the ANN structure is obtained based on experimental knowledge.

Table 1. Input and Output variables of ANN

\begin{tabular}{c|l}
\hline \multicolumn{2}{c}{ Parameters } \\
\hline In_par_1 & Load value in the same day of 5 months before \\
In_par_2 & Load value in the same day of 4 months before \\
In_par_3 & Load value in the same day of 3 months before \\
In_par_4 & Load value in the same day of 2 months before \\
In_par_5 & Load value in the same day of 1 month before \\
In_par_6 & Load value in 5 days before the forecasted day \\
In_par_7 & Load value in 4 days before the forecasted day \\
In_par_8 & Load value in 3 days before the forecasted day \\
In_par_9 & Load value in 2 days before the forecasted day \\
In_par_10 & Load value in 1 day before the forecasted day \\
Out_par & Load value of the forecasted day. \\
\hline
\end{tabular}

In order to compare the performance among ANN traditional method, PSO evolutionary-based algorithm, and proposed ANN combined with modified Bat algorithm, Table 2 summarized the relative error $\sigma_{i}$ and calculate the MAPE percentage. By comparing the results of Table 2, it can be observed that the relative error has decreased in proposed ANN-modified Bat algorithm. The maximum value of relative error in ANN-modified Bat algorithm is 5.8929 which is lower compared to the maximum relative error value for either ANN traditional model and PSO-ANN: 8.4535 and 7.4459 , respectively.

Figure 2. depicts the relative error for 30 predicted ahead days for traditional ANN, ANN-PSO, and ANN-Modified Bat Algorithm, respectively. As it is shown in Figure 2, the relative error of proposed combined method is less than two other methods.
Table 2. Comparison of relative error values of Traditional ANN, and ANN PSO, and proposed ANN-modified Bat algorithm for one-month prediction

\begin{tabular}{|c|c|c|c|}
\hline $\begin{array}{c}\text { Predicted } \\
\text { Day }\end{array}$ & $\begin{array}{l}\mathrm{ANN} \\
\sigma_{i} \%\end{array}$ & $\begin{array}{l}\text { ANN- } \\
\text { PSO } \\
\sigma_{i} \%\end{array}$ & $\begin{array}{l}\text { ANN-Modified Bat } \\
\text { algorithm } \sigma_{i} \%\end{array}$ \\
\hline 1 & 2.1321 & 1.7634 & 3.1223 \\
\hline 2 & 7.4456 & 1.7890 & 1.2311 \\
\hline 3 & 52380 & 3.7440 & 2.1784 \\
\hline 4 & 5.9087 & 3.1079 & 2.4120 \\
\hline 5 & 5.1153 & 1.4959 & 1.3422 \\
\hline 6 & 7.8911 & 3.1760 & 2.2512 \\
\hline 7 & 4.7811 & 2.9080 & 3.2510 \\
\hline 8 & 2.2359 & 7.4459 & 1.7812 \\
\hline 9 & 1.7822 & 5.9923 & 0.7690 \\
\hline 10 & 1.2912 & 4.1160 & 0.1023 \\
\hline 11 & 1.9945 & 1.7230 & 0.0072 \\
\hline 12 & 2.3378 & 1.8230 & 2.7801 \\
\hline 13 & 3.7490 & 4.8185 & 0.8410 \\
\hline 14 & 1.7290 & 1.5181 & 5.1206 \\
\hline 15 & 8.1962 & 3.4510 & 1.7120 \\
\hline 16 & 3.5659 & 1.5294 & 0.9821 \\
\hline 17 & 2.5250 & 5.7843 & 0.1383 \\
\hline 18 & 1.5868 & 1.7333 & 1.7912 \\
\hline 19 & 0.2510 & 1.7020 & 0.9400 \\
\hline 20 & 1.9478 & 1.8551 & 1.8865 \\
\hline 21 & 1.0520 & 3.9239 & 5.8929 \\
\hline 22 & 8.4535 & 1.4434 & 1.4612 \\
\hline 23 & 3.2195 & 1.8511 & 0.5880 \\
\hline 24 & 1.7890 & 3.2223 & 1.8641 \\
\hline 25 & 0.5121 & 1.4934 & 0.4194 \\
\hline 26 & 0.0921 & 3.9146 & 3.4626 \\
\hline 27 & 4.7780 & 0.6930 & 2.0022 \\
\hline 28 & 5.8878 & 2.2655 & 5.1521 \\
\hline 29 & 8.2170 & 0.3121 & 2.4232 \\
\hline 30 & 2.9512 & 0.8122 & 5.4828 \\
\hline МAPE\% & 3.6121 & 2.9878 & 2.2114 \\
\hline
\end{tabular}

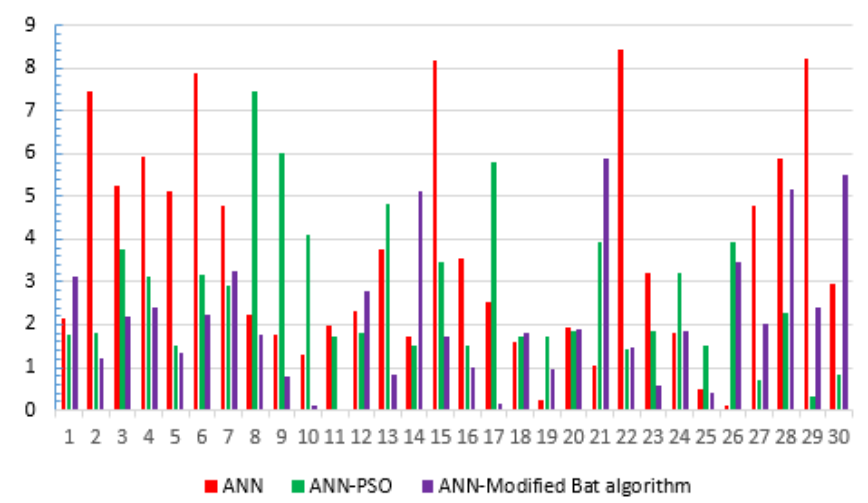

Figure 2. Relative errors of 30 predicted days for ANN, ANNPSO, and ANN-Modified Bat Algorithm. 


\section{CONCLUSION}

A new hybrid method based on traditional ANN for training and tuning the adjusting and weight factors by using modified Bat algorithm is proposed in this study for electric power load forecasting. The superiority of the proposed method is that it leverages the capability of ANN for training and mapping nonlinear relation among variables and by using modified Bat algorithm as an optimization technique to figure out the best values for ANN parameters. In order to demonstrate the superiority of the proposed method, real dataset of electric load consumption of Sergipe province in Brazil is applied. By combining the proposed method with ANN, the performance is enhanced in terms of avoiding the local optima and immature convergence.

\section{REFERENCES}

[1] Reza Yousefian, Amireza Sahami, Sukumar Kamalasadan, Hybrid Transient Energy Function Based Real-Time Optimal Wide-Area Damping Controller, IEEE Trans. Industry Applications, vol. 53, no. 2, March-April, pp. 1506-1516, 2017.

[2] Kavousi-Fard, Abdollah, A Novel Probabilistic Method to Model the Uncertainty of Tidal Prediction, IEEE Trans. Geo. and Remote Sens. 55 (2), 828-833, 2017.

[3] Sahami Amirreza, Shahram Montaser Kouhsari, Making a Dynamic Interaction Between Two-power System Analysis Software, North American Power Symposium (NAPS) 2017, 1-7.

[4] S. Geraeea, M. Shafieib, A. R. Sahamic, S. Alavi, Position Sensorless and Adaptive Speed Design for Controlling Brushless DC Motor Drives, North American Power Symposium (NAPS) 2017, 12-18.

[5] Kavousi-Fard, Abdollah, Modeling Uncertainty in Tidal Current Forecast Using Prediction Interval-Based SVR, IEEE Trans. Sustainable Energy. vol. 8, 2017, 708-715.

[6] Reza Yousefian; Amirreza Sahami; Sukumar Kamalasadan, Hybrid energy function based realtime optimal wide-area transient stability controller for power system stability, IEEE Industry Applications Society Annual Meeting, 2015, pp. $1-8$.

[7] Hsu, Yuan-Yih, and Chien-Chun Yang. "Electrical load forecasting." In Applications of Neural Networks, pp. 157-189. Springer US, 1995.

[8] Papari, Behnaz, Chris S. Edrington, Indranil Bhattacharya, and Ghadir Radman. "Effective Energy Management of Hybrid AC-DC Microgrids with Storage Devices." IEEE Transactions on Smart Grid (2017).

[9] Vu, Tuyen V., Dallas Perkins, Behnaz Papari, Hesan Vahedi, and Chris S. Edrington. "Distributed adaptive control design for cluster of converters in DC distribution systems." In DC Microgrids (ICDCM), 2017 IEEE Second International Conference on, pp. 197-201. IEEE, 2017.

[10] Papari, B., C. S. Edrington, T. V. Vu, and F. DiazFranco. "A heuristic method for optimal energy management of DC microgrid." In DC Microgrids (ICDCM), 2017 IEEE Second International Conference on, pp. 337-343. IEEE, 2017.
[11] Papari, Behnaz, Chris S. Edrington, and Farzaneh Kavousi-Fard. "An Effective Fuzzy Feature Selection and Prediction Method for Modeling Tidal Current: A Case of Persian Gulf." IEEE Transactions on Geoscience and Remote Sensing 55, no. 9 (2017): 4956-4961.

[12] Zhang, Fu-sheng, Fang Liu, Wen-bin Zhao, Zi-an SUN, and Guang-ying JIANG. "Application of grey Verhulst model in middle and long term load forecasting." Power System Technology 5 (2003): 37-40.

[13] Khalilian, A., Sahamijoo, G., Avatefipour, O., Piltan, F., \& Nasrabad, M. R. S. (2014). Design high efficiencyminimum rule base PID like fuzzy computed torque controller. International Journal of Information Technology and Computer Science (IJITCS), 6(7), 77.

[14] Khalilian, A., Piltan, F., Avatefipour, O., Nasrabad, M. R. S., \& Sahamijoo, G. (2014). Design New Online Tuning Intelligent Chattering Free Fuzzy Compensator. International Journal of Intelligent Systems and Applications, 6(9), 75.

[15] Sahamijoo, G., Avatefipour, O., Nasrabad, M. R. S., Taghavi, M., \& Piltan, F. (2015). Research on minimum intelligent unit for flexible robot. International Journal of Advanced Science and Technology, 80, 79-104.

[16] Mokhtar, M., Piltan, F., Mirshekari, M., Khalilian, A., \& Avatefipour, O. (2014). Design minimum rule-base fuzzy inference nonlinear controller for second order nonlinear system. International Journal of Intelligent Systems and Applications, 6(7), 79.

[17] Mori, Hiroyuki, and Shouichi Urano. "Short-term load forecasting with chaos time series analysis." In Intelligent Systems Applications to Power Systems, 1996. Proceedings, ISAP'96., International Conference on, pp. 133-137. IEEE, 1996.

[18] Vu, Tuyen V., David Gonsoulin, Dallas Perkins, Behnaz Papari, Hesan Vahedi, and Chris S. Edrington. "Distributed control implementation for zonal MVDC ship power systems." In Electric Ship Technologies Symposium (ESTS), 2017 IEEE, pp. 539-543. IEEE, 2017.

[19] Papari, Behnaz, Davud Asemani, and Ali Khakpour. "A wide-band time-interleaved A/D converter for cognitive radio application with adaptive offset correction." In Wireless Advanced (WiAd), 2011, pp. 144-148. IEEE, 2011.

[20] Song, Kyung-Bin, Young-Sik Baek, Dug Hun Hong, and Gilsoo Jang. "Short-term load forecasting for the holidays using fuzzy linear regression method." IEEE transactions on power systems20, no. 1 (2005): 96-101.

[21] Chen, Jiann-Fuh, Wei-Ming Wang, and Chao-Ming Huang. "Analysis of an adaptive time-series autoregressive moving-average (ARMA) model for short-term load forecasting." Electric Power Systems Research 34, no. 3 (1995): 187-196.

[22] Yun, Zhang, Zhou Quan, Sun Caixin, Lei Shaolan, Liu Yuming, and Song Yang. "RBF neural network and ANFIS-based short-term load forecasting approach in real-time price environment." IEEE Transactions on power systems 23 , no. 3 (2008): 853-858.

[23] Avatefipour, O., Piltan, F., Nasrabad, M. R. S., Sahamijoo, G., \& Khalilian, A. (2014). Design New 
Robust Self Tuning Fuzzy Backstopping Methodology. International Journal of Information Engineering and Electronic Business, 6(1), 49.

[24] Shahcheraghi, A., Piltan, F., Mokhtar, M., Avatefipour, O., \& Khalilian, A. (2014). Design a Novel SISO Offline Tuning of Modified PID Fuzzy Sliding Mode Controller. International Journal of Information Technology and Computer Science (IJITCS), 6(2), 72.

[25] Kavousi-Fard, Abdollah, Niknam, Taher, M. FotuhiFiruzabad, A Novel Stochastic Framework based on Cloud Theory and $\Theta$-Modified Bat Algorithm to Solve the Distribution Feeder Reconfiguration, IEEE Trans. Smart Grid 7(2)(2015) 740-750.

[26] Kavousi-Fard, Abdollah, W. Su, A Combined Prognostic Model Based on Machine Learning for Tidal Current Prediction, IEEE Trans. Geo. and Remote Sens vol. 15, no. 6, 2017, pp. 3108-3114.

[27] Kavousi-Fard, Abdollah, A Hybrid Accurate Model for Tidal Current Prediction, IEEE Trans. Geo. and Remote Sens. 55 (1), pp. 112-118, 2017.

[28] Bashir, Z. A., and M. E. El-Hawary. "Applying wavelets to short-term load forecasting using PSO-based neural networks." IEEE Transactions on Power Systems 24, no. 1 (2009): 20-27.

[29] Hong, W. C. (2009). Chaotic particle swarm optimization algorithm in a support vector regression electric load forecasting model. Energy Conversion and Management, 50(1), 105-117.

[30] Yang, Xin-She, and Amir Hossein Gandomi. "Bat algorithm: a novel approach for global engineering optimization." Engineering Computations 29, no. 5 (2012): 464-483

[31] Sathya, M. R., \& Ansari, M. M. T. (2015). Load frequency control using Bat inspired algorithm based dual mode gain scheduling of PI controllers for interconnected power system. International Journal of Electrical Power \& Energy Systems, 64, 365-374. 